\title{
L'italiano in Europa nel Duemila: note su una nuova edizione
}

\section{Daniela Goldin Folena}

PUBBLICATO: 31 MAGGIO 2021

$\Omega$ utto ebbe inizio nel giugno 2020 quando il Presidente del Comitato Nazionale per il centenario della nascita di Gianfranco Folena mi contattò proponendomi un'Introduzione alla ristampa di Litaliano in Europa, certo l'opera più famosa e introvabile dello studioso. La mia risposta fu parzialmente negativa perché pensavo che un'Introduzione ad un libro che aveva già una bellissima Introduzione-Premessa dell'Autore fosse assolutamente inutile. Prospettavo al massimo una Nota redazionale dove avrei ricordato anche i Convegni dedicati in questi decenni proprio a quel libro.

A questo segui il contatto con l'editore Franco Cesati che da molti anni già si era offerto per l'esclusiva di quella che sembrava a prima vista una semplice ristampa, e al quale già in passato avevo fornito un piccolo primo campione di refusi o errori su cui eventualmente intervenire. Così, dall'idea di un'Introduzione si era passati a quella di un controllo completo della ristampa-riedizione. Cosa a cui mi sarei prestata ben volentieri per esperienze simili fatte in passato. Ci fu si un piccolo contrattempo o una, per me risolvibile, 'incomprensione' iniziale con l'editore. Forse perché la sua casa editrice ha particolare consuetudine con edizioni di testi di argomento linguistico, dove prevale la tradizione di suddivisione dei testi in paragrafi e sottoparagrafi con numerazioni e sottonumerazioni, propria anche dei manuali, nella sua prima prova di ristampa l'Indice originale era stato rimodellato sullo schema appunto più frequente di testi di altre tematiche: incolonnamento con numeri progressivi dei titoli dei singoli paragrafi dei capitoli originali e ripetizione degli stessi titoli all'interno dei singoli saggi. Con questo si vanificava l'originalità dell'Indice stesso, su cui per altro aveva già insistito Erasmo Leso nel suo intervento al Convegno proprio della Crusca del 20II ${ }^{[\mathrm{I}]}$. Una funzione aveva pure l'originale anepigrafia dei singoli paragrafi allinterno dei saggi, originariamente marcati solo dalla loro numerazione progressiva; o almeno cosi l'avvertivo io: quella doppia spaziatura tra un paragrafo e l'altro, senza che nell'immediato orizzonte visivo della pagina ci fosse anticipato verbalmente il contenuto del secondo, costituiva una strategica pausa e nello stesso tempo creava suspense, l'attesa di una nuova avventura critica, contigua al testo precedente ma - come sempre ci si aspetta dai testi di Folena - prevedibilmente carica di novità e di sorprese. Per questi motivi, alla proposta iniziale dell'editore avevo risposto drasticamente con una frase per me del tutto inusuale: «Come allieva e come erede, rifiuto questa soluzione lontana dalle intenzioni e dal metodo di Folena.» Il problema si risolse molto facilmente con un incontro l's ottobre 2020 a Bologna, a metà strada tra Padova, dove si stava svolgendo il primo Convegno del centenario, e Firenze, sede della Casa Editrice. Come "buoni argomenti" - per usare le parole di Don Basilio nel Barbiere di Siviglia rossiniano -, a conferma delle mie convinzioni avevo portato con me volumi-raccolte di saggi di Folena ${ }^{[2]}$ da lui stesso pubblicati, e Lingua nuova e antica di Giorgio Pasquali ma curata ancora da Folena ${ }^{[3]}$, dove si proponeva sempre lo schema del nostro volume: mi sembrava che tanto bastasse per confermare l'autenticità di quel sistema di organizzazione editoriale, di un metodo 'illuminante' di predisporre agli occhi del lettore l'itinerario - termine ben caro allo stesso Folena - critico in cui saremmo stati guidati nella lettura $^{[4]}$.

Risolto facilmente il problema Indice, l'editore mi propose quindi di rivedere la nuova stampa consegnandomi in quell'occasione il nuovo volume, nel quale aveva già adottato non solo le correzioni ai refusi segnalati anni prima, ma soprattutto le correzioni (in parte le stesse già segnalate) trovate in 
un foglio di Errata corrige, battuto con una mitica Olivetti Lettera 22 (nel 1983 si era ben lontani dai computer) dallo stesso Folena che aveva giustamente previsto una seconda edizione per un suo volume andato pressoché a ruba fin dalla sua uscita. Eravamo insomma tutti e due convinti che la mia rilettura sarebbe stata un piacevole ripercorrere pagine note, da fare nel breve arco delle tre settimane concessemi. E invece... Avevo cominciato la lettura dall'argomento a me più familiare delle lettere di Mozart e del suo italiano. Ma il controllo con gli originali mozartiani doveva riservarmi parecchie sorprese, cosi come quello dei Mémoires di Goldoni e delle lettere di Voltaire, per il numero imprevisto di imperfezioni soprattutto nelle citazioni. Solo dopo i controlli a quei saggi conclusivi, mi decisi a riprendere in mano il volume dall'inizio. Col risultato di essere presa ancor più dall'entusiasmo per un'opera che appare ancora nuova, e col pericolo che proprio quell'entusiasmo compromettesse la lucidità della mia revisione di correttore di bozze: avrei dovuto, parafrasando un discusso principio della filologia, emendare sine interpretatione, proseguire una asettica lettura tutta concentrata sulle singole parole, anzi sui singoli simboli e caratteri. Ma ovviamente, come già mi era successo nelle esperienze simili - Scrittori e scritture e altro - , il piacere, anche, di ripercorrere i ragionamenti dell'autore, di riflettere sui singoli passi dei testi mi imponeva pause, direi di ammirazione, come se fossi alla prima lettura.

Perché la lettura continua e progressiva del volume a distanza di quasi 4 decenni, con ovviamente anche anni di esperienza critica personale, non poteva che farmi recepire quel testo in una prospettiva e con occhi diversi. Colpiscono ancora la densità delle argomentazioni, una straordinaria padronanza delle letterature primarie e secondarie (complice anche la sua mitica memoria), un orizzonte europeo foltissimo di autori 'antichi' e di critici moderni, quale frutto di una frequentazione assidua - e particolarmente produttiva - di biblioteche italiane ed europee ma anche nordamericane (siamo ben lontani dall'epoca in cui i maggiori critici potevano permettersi di consultare a casa propria persino i manoscritti); colpisce poi la capacità di tirare le fila e di far convergere argomenti che sembrerebbero portare in direzioni diverse, tutti tesi invece alla plausibilissima conferma di tesi nuove e originali. Ma è stato il capitolo su Goldoni a sorprendermi come non immaginavo, proprio uno degli autori che io stessa ho sempre continuato a frequentare, con l'occhio per altro rivolto ad una bibliografia dove il nome di Folena aveva una posizione preminente. Ma solo in questa occasione ho percepito come mai prima la singolarità dell'atteggiamento proprio dell'autore Folena verso l'autore oggetto delle sue indagini. I saggi su Goldoni sono tutti entro gli anni '5o del secolo scorso, vale a dire nella stagione dei suoi zo anni, a partire dal suo trasferimento definitivo a Padova. Vero che secondo l'aneddotica da lui stesso diffusa anche oralmente, proprio durante i suoi primi viaggi verso la provincia veneta Folena concepi per la prima volta l'idea di dedicarsi all'autore veneziano e addirittura di fornire a studiosi italiani e francesi, non più che agli operatori del teatro, uno strumento lessicografico, fino ad allora inesistente, utile alla comprensione della lingua madre del nostro massimo commediografo. Sarà stato questo singolare afflato nei confronti di un autore pressoché inedito, certo molto poco considerato dalla cultura toscanocentrica, o forse più sarà stato l'entusiasmo per un personaggio che univa ad uno straordinario senso del teatro un suo originalissimo approccio anzi uso della letteratura, che veniva da una trasparente sensibilità primaria verso il pubblico e una profonda generosità mentale, se così si può dire, verso il complesso popolo dei teatranti che le sue commedie eseguivano (nei Mémoires colpisce sempre quel mettersi sempre dietro le quinte di Goldoni per godere e compiacersi più del successo delle compagnie teatrali che dei propri testi), e infine sarà stato quell'esilio in parte cercato tra la haute culturale parigina che però non poteva soddisfare completamente le sue ambizioni; tutti questi elementi, nei quali ancora oggi noi possiamo individuare una sorta di affinità con il carattere e le attitudini comunicative, nel senso migliore del termine, dello stesso Folena spiegano un carattere peculiare dei suoi saggi goldoniani, quale ho avvertito proprio in occasione di questa loro lettura sistematica e continua, il suo inedito atteggiamento di sim-patia verso l'oggetto della sua indagine, in 
sintesi, arriverei a dire, una 'passione' che va ben al di là dell'analisi formale (ma la 'forma' non è mai l'oggetto primario e finale delle analisi di Folena) dei testi di Goldoni, dei loro meccanismi, e dei contesti nei quali sono nati.

Si capisce come per me sia stato difficile leggere il riproposto Litaliano in Europa con totale neutralità quale si richiederebbe a un 'professionale' curatore di volumi. E non escludo mi siano sfuggiti ancora errori e refusi. Ma posso testimoniare il mio impegno ricordando che delle 520 pagine che formano la nuova stampa ci sono tracce della mia lettura attenta anche a dettagli minimi su almeno 500. Qualcuno si chiederà: Possibili tanti errori di Folena? Errori ed aggiustamenti minimi furono avvertiti e corretti da lui subito dopo la prima edizione, come si vede dalla riproduzione a stampa del foglio di errata corrige in questa seconda. Ma la correzione di refusi e sviste non era certo la priorità di Folena, preso comera dalla sua molteplice intensa attività didattica e poi da quella, pure a vasto raggio, organizzativa nel senso più ampio della parola. Alla fine della sua Premessa si legge il suo ringraziamento ad alcuni, diciamo, giovani studiosi, per lo più suoi scolari. Posso testimoniare, per essere in quel breve elenco, che nessuno di noi è mai stato ingaggiato quale correttore di bozze: un 'favore' o peggio un servizio che il nostro comune maestro non ha mai preteso da chicchessia. Quei ringraziamenti alludono con estrema generosità a minimi suggerimenti, a segnalazioni di cose che potevano interessare la sua ricerca (personalmente, quella di alcune lettere italiane di Mozart, da me allora particolarmente frequentate per le mie ricerche sulle opere di Mozart e di Da Ponte).

Giunta al fine... Credo dobbiamo sostanzialmente questo riproposto L'italiano in Europa alla sana ostinazione dell'editore Franco Cesati, al suo sincero credere in un volume esemplare e ancora attualissimo, tanto da superare esitazioni, tentennamenti (altrui) e anche ostacoli che fino a pochissimo tempo fa gli impedivano la realizzazione del suo progetto. A lui vanno i ringraziamenti convintissimi miei e credo di tutti gli studiosi che conoscono il valore di questa come di tutte le opere di Gianfranco Folena.

Note:

I. Il Convegno, svoltosi a Firenze il 6-7 maggio 20II, era proprio intitolato Litaliano in Europa. Gli Atti, a cura di N. Maraschio, D. De Martino, G. Stanchina, pubblicati pure dall'Accademia della Crusca, sono usciti a Firenze nel 20I2. Il saggio di E. Leso, Litaliano in Europa trent'anni dopo, pp. II25, contiene osservazioni su tutti gli Indici del volume alle pp. I2-3. Con altre argomentazioni ne ho parlato anch'io nella Premessa alla seconda edizione del volume foleniano.

2. Cfr. Culture e lingue del Veneto medievale, Padova, Editoriale Programma, I99o, e Il linguaggio del caos. Studi sul plurilinguismo dialettale, Torino, Bollati Boringhieri, I99I.

3. G. Pasquali, Lingua nuova e antica. Saggi e note, a cura di G. Folena, Seconda edizione, Firenze, Felice Le Monnier, 1985 (la prima edizione di Pasquali era uscita nel I963).

4. Un modello che nel mio piccolo e nei limiti del materiale originale disponibile ho cercato di rispettare nella curatela di altri volumi postumi foleniani, distribuendo almeno la materia in parti e capitoli distinti: cfr. G. Folena, Scrittori e scritture. Le occasioni della critica, Introduzione di M. Berengo, Edizione a cura di D. Goldin Folena, Bologna, Il Mulino 1997 (il titolo non è originale, ma ricavato fedelmente dai progetti editoriali di Folena); Id., Textus testis. Lingua e cultura poetica delle origini, Torino, Bollati Boringhieri, 2002. Anche se con riduzioni e manomissioni indebite di terzi, i curatori 
sono da individuare nella sottoscrizione dell'Avvertenza editoriale (originariamente, Nota redazionale), cioè D. Goldin Folena e G. Peron.

\section{Cita come:}

Daniela Goldin Folena, L'italiano in Europa nel Duemila: note su una nuova edizione, “Italiano digitale", XVII, 2021/2 (aprile-giugno)

DOI: $10.35948 / 2532-9006 / 2021.7530$

Copyright 2021 Accademia della Crusca

Pubblicato con licenza creative commons CC BY-NC-ND 